\title{
The Possibility of Estimating the Coseismic Deformation from Water Level Observations in Wells
}

\author{
G. N. Kopylova ${ }^{a}$, G. M. Steblov ${ }^{b, c}$, S. V. Boldina ${ }^{a}$, and I. A. Sdel'nikova ${ }^{c}$ \\ ${ }^{a}$ Kamchatka Branch of the Geophysical Service, Russian Academy of Sciences, \\ bulv. Piipa 9, Petropavlovsk-Kamchatskii, 883006 Russia \\ ${ }^{b}$ Schmidt Institute of Physics of the Earth, Russian Academy of Sciences, \\ Bol'shaya Gruzinskaya ul. 10, Moscow, 123995 Russia \\ ${ }^{c}$ Geophysical Service, Russian Academy of Sciences, prosp. Lenina 189, Obninsk, 249030 Russia \\ Received December 9, 2008
}

\begin{abstract}
A method for point estimation of coseismic volume deformation from amplitudes of earthquakegenerated changes in the water level and from the tidal sensitivity of the water level is presented. Application of this method is illustrated by six Kamchatka earthquakes of 1997-2004 with $m_{w}=6.2-7.8$ that occurred $128-316 \mathrm{~km}$ from the YuZ-5 well. Estimates of coseismic deformation provided by the level gage observations and by the model agree in amplitude and sign. The model reproduces an extended dislocation source in a homogeneous elastic isotropic half-space. Source rupture parameters correspond to focal mechanisms from the CMT international catalog. Consistence in experimental and theoretical estimates of the volume coseismic deformation indicates the high strain-resistance properties of YuZ-5 borehole and the possibility of quantitative estimation of seismotectonic deformation from high-precision water level measurements with time resolution not lower than 10 minutes.
\end{abstract}

DOI: $10.1134 / \mathrm{S} 1069351310010040$

\section{INTRODUCTION}

Water level observations in boreholes are a method for the geophysical monitoring of the seismically active regions and for the search for earthquake precursors [Kissin, 1993]. The water level sensitivity to changes in the stress-strain state of the medium is controlled by the deformation of water-bearing rocks and variations in the pore pressure during the processes of earthquake preparation, development of seismic dislocations, and seismic wave propagation. Earthquake impacts on the boreholereservoir system may manifest themselves as different types of water level variations corresponding to a static change in the stress state of water-bearing rocks during the formation of ruptures in sources as well as to the passage of seismic waves and earthquake preparation processes [Kopylova, 2006].

Formation of ruptures in the sources of strong earthquakes is accompanied by changes in the static stress state of confined ground water reservoirs at distances up to dozens-a few hundreds of kilometers. Such an effect appears as coseismic step-like rises or drawdowns of the water level in boreholes, which are observed within several seconds (tens of seconds) after the rupture formation and which reflect the pore-pressure response to the elastic deformation of water-bearing rocks. As was shown in [Wakita, 1975], the areal distribution of the zones of coseismic compression and extension and, consequently, the zones of water level changes (rising or falling) in wells are controlled mainly by the focal mechanisms of earthquakes. Amplitudes of coseismic step-like changes in the water level usually vary from tenths to a few dozens of centimeters.

A necessary condition for detecting the coseismic water level changes is a sufficiently high sampling rate of measurements, not lower than one reading per 10$15 \mathrm{~min}$. If the water level observations are made at a lower sampling rate, e.g., one reading per hour, the coseismic changes cannot be revealed due to their relatively small amplitudes and short times of development, or due to their masking by superimposed longer-duration and higher-amplitude effects caused by the passage of seismic waves related mostly to the changes in the properties of the water-permeability of the borehole-reservoir system. The latter circumstance, i.e., nearly simultaneous action of two factors of seismicity on the state of the boreholereservoir system (changes in the static stress state of the groundwater reservoir and the dynamic action of seismic waves) complicates the identification of coseismic changes in the water level and often leads to false conclusions as to their amplitudes, occurrence times, and, correspondingly, their relevance to the focal parameters of the earthquake.

The emergence of coseismic step-like changes and the presence of a tidal component in the water level variations in a particular borehole can be considered as evidence of its strain sensitivity and informativeness regarding the geophysical monitoring of seismically active regions aimed at the detection of seismotectonic signals, including hydrodynamic earthquake precursors [Kissin, 
1993; Kopylova and Boldina, 2006; Roeloffs, 1988; Rojstaczer, 1988; Rojstaczer and Agnew, 1989].

the tidal impact resulting in changes in the stressstrain state of the Earth's crust is generated by gravitational interaction in the Earth-Moon-Sun system. This impact is fairly well studied and can now be calculated for any arbitrary point on the Earth's surface with an accuracy of not lower than $50 \%$ compared to the data of instrumental measurements [Melchior, 1968]. A certain discrepancy between theoretical estimates and instrumental measurements of tidal deformations on the Earth's surface may be explained by the presence of local gravity inhomogeneities at the observation points as well as by the influence of the oceanic tide that includes waves that are similar to the Earth tidal waves in periods but different in phases .

Quantitative estimation of the parameters characterizing the tidal response of the water level in a well, such as amplitudes of particular tidal waves and differences between the phases of tidal waves in water-level measurements and the corresponding phases of the tidal potential, makes it possible to obtain the tidal sensitivity of water level, which can be used as a normalizing factor in estimating the coseismic deformation in the region of a borehole [Kopylova and Boldina, 2006; Roeloffs, 1988; Rojstaczer, 1988]. In case of a static confined response of the water level in the borehole to the changes in the stress-strain state of the reservoir, which allows us to disregard the groundwater flow (filtration) and the inertia in the water exchange between the borehole and the reservoir, the tidal sensitivity is strongly controlled by elastic parameters of the reservoir [Kopylova and Boldina, 2006; Rojstaczer and Agnew, 1989]. In case of a strong distortion in the poroelastic response of the water level to the Earth tides and coseismic deformation, neither tidal variations nor coseismic step-like changes in the water level variations are detected (for example, in boreholes opening the free surface groundwaters), and such boreholes are not informative for the quantitative estimation of a seismotectonic deformation. It hence follows that the occurrence of coseismic step-like changes in the water level is important evidence for the strain sensitivity of the water level in certain wells.

The YuZ-5 well $\left(53.17^{\circ} \mathrm{N}, 158.41^{\circ} \mathrm{E}\right.$, depth $800 \mathrm{~m}$, water level at a depth of $1.5 \mathrm{~m}$ ) (Fig. 1) opens high-pressure groundwater in Late Cretaceous metamorphized aleurolites and schists in the interval $310-800 \mathrm{~m}$. Water level and air pressure measurements with 10 min time resolution have been carried out in this borehole since September 1997 [Kopylova, 2006]. Observations are conducted by the Kamchatka Branch of the Geophysical Service of the Russian Academy of Sciences (KF GS RAN). Throughout the period of observation, the accuracies of recording variations in the water level and air pressure were $\pm 0.1 \mathrm{~cm}$ and $\pm 0.2 \mathrm{hPa}$, respectively.

Based on the data on the water level response to the air pressure variations, a static confined barometric water response to the changes in the stress-strain state of the medium has been detected in the YuZ-5 borehole in peri- ods ranging from hours to a few dozen days. An undistorted tidal response of the water level is found for this borehole at periods of tidal waves corresponding to semidiurnal and diurnal groups [Kopylova and Boldina, 2006].

In 1997-2004, six coseismic step-like changes with amplitudes of $0.25-12.0 \mathrm{~cm}$ were observed in the water level variations in the YuZ-5 well. These jumps occurred during local earthquakes with $M=6.2-7.8$ that hit at distances $R=128-316 \mathrm{~km}$ from their instrumental hypocenters (Fig. 1, Table 1). These jumps in the water level were detected mainly within the 10-min interval including the occurrence time of the earthquake. Four cases of a drop in the water and two cases of a rise in the water were observed [Kopylova. 2006]. The maximum amplitude of the coseismic water-level drop $(\Delta h=-12 \mathrm{~cm})$ was observed during the Kronotskii earthquake of December 5, 1997, with $M_{w}=7.8$ (no. 1 in Table 1 and Fig. 1). Consequently, three types of water level variations caused by the earthquake were revealed to have occurred. These variation types corresponded to a hydrogeodynamic precursor, a coseismic step-like change, and a prolonged postseismic lowering and restoration [Kopylova, 2006].

In this work, we estimate the volume coseismic deformation of the water-bearing rocks in the region of the YuZ-5 borehole during six earthquakes (Fig. 1, Table 1). These estimates are derived from the amplitudes of coseismic jumps with the use of the calculated water level tidal sensitivity to the expected volume deformation $\left(A_{V}\right)$ obtained from the tidal analysis of hourly average water level variations. For comparison, we present the theoretical estimates of the volume coseismic deformation in the region of the YuZ-5 borehole for all of the six earthquakes, which are calculated from the dislocation model in a uniform elastic isotropic half-space [Okada, 1985]. For determining the rupture parameters in seismic sources we used the focal mechanisms from the Global CMT international catalog (http://www.globalcmt.org/). With the use of the above mentioned model and given focal mechanisms, we constructed horizontal distributions of the volume coseismic deformation at a depth of $500 \mathrm{~m}$ and estimated the relevant quantities in the region of the YuZ-5 borehole. Calculations were conducted by the GS (Geophysical Survey) of the Russian Academy of Sciences, Sector of Geodynamic Monitoring.

\section{ESTIMATION OF THE COSEISMIC DEFORMATION FROM WATER LEVELOBSERVATIONS}

An example of 10-min records of the water level and air pressure during the Kronotskii earthquake of December 5, 1997 is presented in Fig. 2a. It is seen that the coseismic step-like drop with an amplitude of $\Delta h=-$ $12 \mathrm{~cm}$ ( 1 in Fig. 1b) is masked largely by barometric tidal variations in the water level, as well as by the development of a long-lasting postseismic lowering, which immediately followed the jump in the water level. 

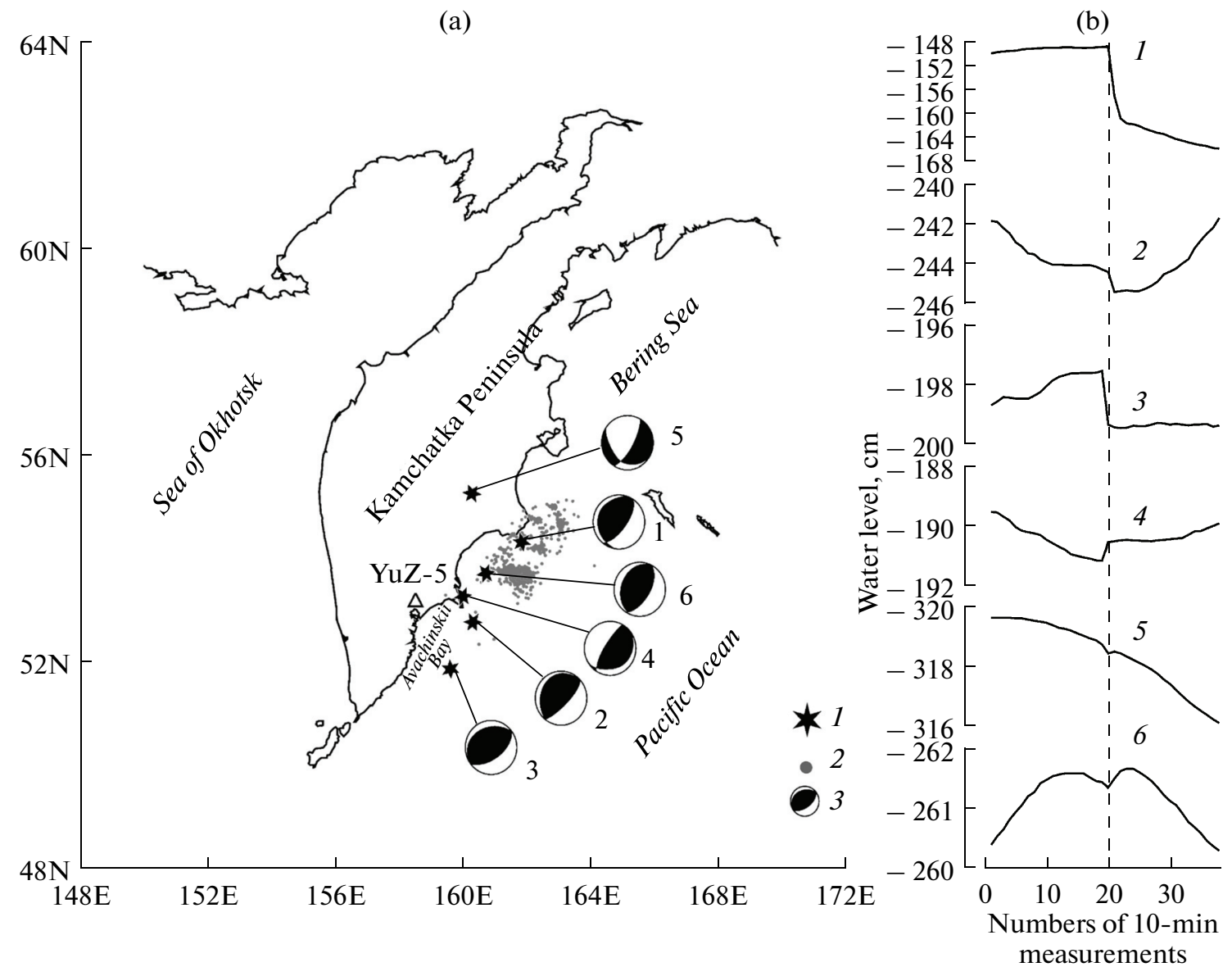

Fig. 1. (a) Schematic positions of the YuZ-5 borehole, epicenters of earthquakes 1-6 (Table 1), and the source of the December 5, 1997, Kronotskii earthquake with $M_{w}=7.8$ from aftershocks of the first day: (1) earthquake epicenters; (2) epicenters of aftershocks of the Kronotskii earthquake from December 5 to December 12, 1997; (3) diagrams of focal mechanisms of CMT earthquakes, and (b) coseismic water level changes recorded during earthquakes 1-6 (see Table 1). The numbers of 10-min water level measurements, including 19 measurements before earthquakes and 19, after, are indicated on the horizontal axis.

Figure $2 \mathrm{~b}$ shows the fragment of hourly average observational data used for estimating the tidal response of the water level. Only barometric and tidal variations, the seasonal trend, and a weak response to the atmospheric precipitation are revealed in the water-level variations from May 26 through November 5, 2003. No coseismic steplike changes or other water level variations caused by earthquakes were observed in this period.

The barometric response of the water level in the YuZ-5 borehole was estimated from the behavior of the amplitude transfer function that relates the air -pressure variations to the water level variations. Within the period range from $6 \mathrm{~h}$ to a few days, this response shows a constant barometric efficiency $E_{b}=\Delta h_{a} / \Delta p_{a}=0.39 \mathrm{~cm} / \mathrm{hPa}$, where $\Delta h_{a}$ is the amplitude of barometric variations in the water level (cm), and $\Delta p_{a}$ is the amplitude of changes in the air pressure (hPa) [Kopylova, 2006]. The phase difference between variations in the water level and the air pressure in this range of periods is close to $-180^{\circ}$, which indicates that the groundwater reservoir is isolated quite well by the overlying rocks, and that the water-level response to pore-pressure variations during the elastic deformation of water-bearing rocks is not distorted. The hypothesis of a static confined response of the water level to the changes in the stress-strain state of water-bearing rocks is assumed for YuZ-5 borehole in this range of periods [Kopylova and Boldina, 2006; Roeloffs, 1988; Rojstaczer, 1988; Rojstaczer and Agnew, 1989]. At shorter periods (2-6h), the $E_{b}$ value increases from 0.2 to $0.39 \mathrm{~cm} / \mathrm{hPa}$, which is caused mainly by the inertial character of the water exchange between the borehole and the saturated water-bearing rocks [Boldina and Kopylova, 2006].

Parameters of the tidal response of the water level (the amplitude of the tidal waves, the amplitude factors being the ratio of the particular wave amplitudes identified in the water-level variations to the corresponding values of theoretical volume deformation; and phase differences between the tidal waves in water-level variations and the corresponding phases of the tidal potential) were estimated with the use of the ETERNA 3.0 program [Wenzel, 1994]. The results are presented in Table 2. 
Table 1. Parameters of earthquakes and coseismic water level changes in the YuZ-5well, source rupture parameters and estimates of coseismic deformation

\begin{tabular}{|c|c|c|c|c|c|c|c|c|c|c|c|c|c|}
\hline \multirow[b]{2}{*}{$\dot{\mathbf{z}}$} & \multirow[b]{2}{*}{ 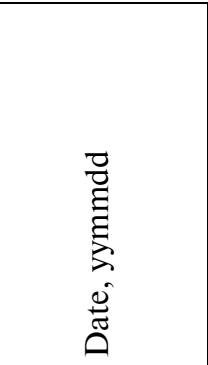 } & \multirow[b]{2}{*}{ 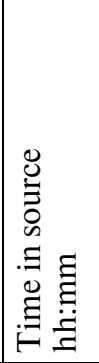 } & \multicolumn{2}{|c|}{$\begin{array}{c}\text { Coordinates, } \\
\text { deg }\end{array}$} & \multirow[b]{2}{*}{ 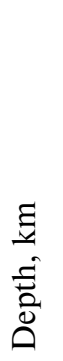 } & \multirow[b]{2}{*}{$z^{3}$} & \multirow{2}{*}{ 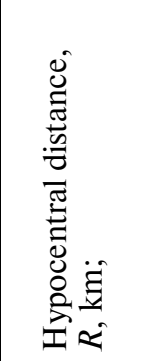 } & \multirow{2}{*}{ 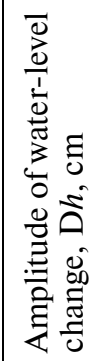 } & \multicolumn{3}{|c|}{ Source parameters, deg } & \multicolumn{2}{|c|}{$\begin{array}{l}\text { Volume coseismic defor- } \\
\text { mation in the region } \\
\text { of YuZ- } 5 \text { borehole, } 10^{-9}\end{array}$} \\
\hline & & & $\mathrm{N}$ & $\mathrm{E}$ & & & & & $\begin{array}{c}\text { strike az- } \\
\text { imuth of } \\
\text { rupture } \\
\text { plane }\end{array}$ & $\begin{array}{l}\text { dip an- } \\
\text { gle of } \\
\text { rupture } \\
\text { plane }\end{array}$ & $\begin{array}{l}\text { direc- } \\
\text { tion of } \\
\text { move- } \\
\text { ment }\end{array}$ & $\begin{array}{c}\text { from coseis- } \\
\text { mic changes } \\
\text { in water lev- } \\
\text { el, } D_{1}\end{array}$ & $\begin{array}{l}\text { from mod- } \\
\text { el of dislo- } \\
\text { cation } \\
\text { source, } D_{2}\end{array}$ \\
\hline 1 & $\begin{array}{l}\text { December 5, } \\
1997\end{array}$ & $11: 27$ & 54.0 & 162.3 & 25 & 7.8 & $316.200 *$ & -12.0 & 202 & 23 & 74 & $75 \pm 4$ & 15.5 \\
\hline 2 & June 1, 1998 & $05: 34$ & 52.81 & 160.37 & 31 & 6.9 & 140 & -1.0 & 210 & 22 & 78 & $6.3 \pm 0.7$ & -2.4 \\
\hline 3 & March 8, 1999 & $12: 26$ & 51.93 & 159.72 & 7 & 7.0 & 164 & -1.7 & 242 & 28 & 101 & $10.6 \pm 0.8$ & 43.2 \\
\hline 4 & $\begin{array}{l}\text { December 20, } \\
2000\end{array}$ & 09:20 & 53.31 & 160.06 & 65 & 6.2 & 128 & 0.6 & 220 & 69 & 77 & $-3.8 \pm 0.5$ & -0.4 \\
\hline 5 & June 16, 2003 & $22: 08$ & 55.30 & 160.34 & 190 & 6.9 & 328 & -0.3 & 123 & 32 & -161 & $1.9 \pm 0.4$ & 0.9 \\
\hline 6 & $\begin{array}{l}\text { March 20, } \\
2004\end{array}$ & 08:53 & 53.74 & 160.76 & 31 & 6.2 & 169 & 0.25 & 216 & 34 & 90 & $-1.6 \pm 0.4$ & -0.5 \\
\hline
\end{tabular}

Note: * The hypocentral distance from borehole YuZ-5 to the midpoint of the displacement plane

For eight waves of the diurnal and semidiurnal groups $\left(Q_{1}, O_{1}, M_{1}, J_{1}, 2 N_{2}, N_{2}, M_{2}, S_{2} K_{2}\right)$, the tidal parameters are determined quite reliably from the level-gage data at the signal-to-noise ratio $\geq 10(12-272)$. The tidal parameters for the most intense wave $P_{1} S_{1}$ were disregarded due to the noises of water level variations by meteorological factors in a diurnal period. The amplitude factors for the selected eight waves lie within the range 0.138$0.216 \mathrm{~cm} / 10^{-9}$. Distinctions in amplitude factors for particular waves are probably due to the specific features in formation of the tidal response in different frequency ranges, as well as to a certain distortion of tidal amplitudes in water-level variations caused by the oceanic tide, which contains waves of the same periods but different phases [Kopylova and Boldina, 2006]. In particular, a certain influence of the oceanic tide is evidenced by the phase difference between tidal variations in the water level and the corresponding components of the theoretical earth tide, which are equal to $-154^{\circ} \ldots-179^{\circ}$ and differ from the "perfect" value $\left(-180^{\circ}\right)$ by $10^{\circ}$ on average .

The linear dependence of the amplitudes of tidal waves identified in the water-level variations $H_{t}(\mathrm{~cm})$ on the theoretical amplitudes of volume deformation $D_{t}$, calculated in accordance with the CTED model of tidal potential [Wenzel, 1994] is presented in Fig. 3. A close linear relationship is observed between these quantities, which has the following form in the $95 \%$ confidence interval:

$$
H_{t}=(0.161 \pm 0.008) D_{t}+(0.004 \pm 0.068) \text {. }
$$

The slope of the linear relation between the identified amplitudes in tidal water level variations and the corresponding values of volume deformation $\Delta H_{t} / \Delta D_{t}=A_{V}$ is $\approx 0.161 \mathrm{~cm} / 10^{-9}$. This value seems most suitable as the characteristic of tidal sensitivity of the water level, $A_{\mathrm{v}}$, for the YuZ-5 borehole in the entire range of tidal periods.

Based on the assumption [Kopylova and Boldina, 2006; Rojstaczer, 1988] of the existence of a linear relationship between the water-level variations and the volume deformation of water-bearing rocks within at least three orders of magnitude $\left(10^{-9}-10^{-7}\right)$, the seismotectonic deformation can be estimated from the amplitudes of water-level variations in the range of periods where a static confined response occurs, by the formula:

$$
D=-\Delta h / A_{\mathrm{v}},
$$

where $D$ is the volume deformation in units of $10^{-9}$, with positive values corresponding to extension and negative, to compression; $\Delta h$ is the amplitude of water-level variations in $\mathrm{cm}$, with a positive value corresponding to a rise in the water-level, and a negative value, to a fall; and $A_{V}$ is the tidal sensitivity of the water level in $\mathrm{cm} / 10^{-9}$.

The accuracy of recording the water-level variations in the YuZ-5 borehole is $0.1 \mathrm{~cm}$; therefore, if $A_{V}=$ $0.161 \mathrm{~cm} / 10^{-9}$, appreciable water level variations can be expected for the volume deformation of the opened groundwater reservoir with amplitudes of at least a few units of $10^{-9}$.

The estimates of the coseismic deformation in the region of the YuZ-5 borehole, taking into account the $95 \%$ confidence interval in dependence (1), are presented in Fig. 3a for five earthquakes (nos. 2-6, Table 1.) The corresponding estimate for the Kronotskii earthquake (no. 1, Table 1) is shown in Fig. 3 b. 

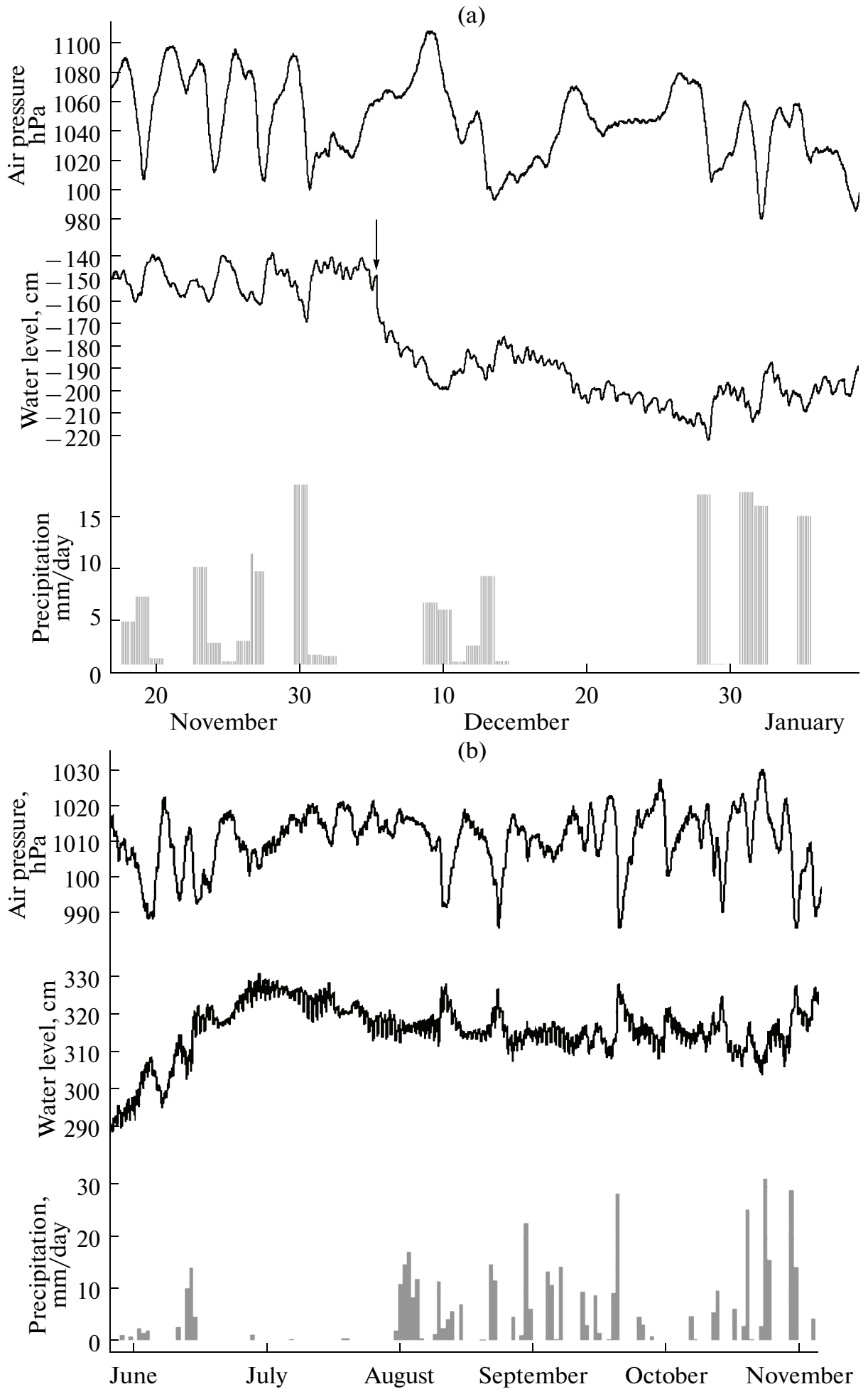

Fig. 2. Observational data on the water level and air pressure variations in the YuZ-5 borehole compared to diurnal sums of precipitation: (a) 10-min records from November 17, 1997 through January 8, 1998 during the Kronotskii earthquake (marked by the black arrow); and (b) hourly mean observations of the air pressure and water level from May 26 through November 5, 2003.

\section{ESTIMATION OF COSEISMIC DEFORMATION FROM THE MODEL OF DISLOCATION}

The volume coseismic deformation in the vicinity of IZVESTIYA, PHYSICS OF THE SOLID EARTH Vol. 46 the YuZ-5 borehole was estimated for all of the six earthquakes in accordance with the model of the dislocation in a homogeneous elastic isotropic half-space. The program developed by Y. Okada, on the basis of analytical expres- 
Table 2. Results of tidal analysis of water-level variations in YuZ-5 borehole with the use of ETERNA 3.0 program [Wenzel, 1994]

\begin{tabular}{|c|c|c|c|c|c|}
\hline Wave, period, $\mathrm{h}$ & $\begin{array}{l}\text { Amplitude of volume } \\
\text { deformation } D_{t}, 10^{-9} \text { units }\end{array}$ & $\begin{array}{l}\text { Amplitude of water } \\
\text { level } H_{t}, \mathrm{~cm}\end{array}$ & Signal-to-noise & $\begin{array}{l}\text { Amplitude factor, } \\
\mathrm{cm} / 10^{-9}\end{array}$ & Phase shift, deg \\
\hline$Q_{1} 26.87$ & 1.40 & 0.276 & 36 & $0.197 \pm 0.005$ & $-166 \pm 2$ \\
\hline$O_{1} 25.82$ & 7.31 & 1.338 & 174 & $0.183 \pm 0.001$ & $-166 \pm 0.3$ \\
\hline$M_{1} 24.83$ & 0.57 & 0.102 & 13 & $0.178 \pm 0.013$ & $-168 \pm 4$ \\
\hline$P_{1} S_{1} 23.93$ & 10.28 & 1.161 & 151 & $0.113 \pm 0.001$ & $-170 \pm 0.4$ \\
\hline$J_{1} 23.10$ & 0.57 & 0.094 & 12 & $0.163 \pm 0.013$ & $-179 \pm 4$ \\
\hline$O O_{1} 22.31$ & 0.31 & 0.053 & 7 & $0.169 \pm 0.024$ & $-174 \pm 8$ \\
\hline $2 N_{2} 12.87$ & 0.21 & 0.045 & 13 & $0.216 \pm 0.017$ & $-177 \pm 4$ \\
\hline$N_{2} 12.66$ & 1.30 & 0.220 & 64 & $0.170 \pm 0.003$ & $-177 \pm 1$ \\
\hline$M_{2} 12.42$ & 6.78 & 0.940 & 272 & $0.138 \pm 0.001$ & $-172 \pm 0.2$ \\
\hline$L_{2} 12.19$ & 0.19 & 0.028 & 8 & $0.147 \pm 0.018$ & $-168 \pm 7$ \\
\hline$S_{2} K_{2} 12.00$ & 3.16 & 0.457 & 132 & $0.145 \pm 0.001$ & $-154 \pm 0.4$ \\
\hline$M_{3} 8.28$ & 0.03 & 0.008 & 3 & $0.273 \pm 0.086$ & $-138 \pm 18$ \\
\hline
\end{tabular}

Note: The waves whose parameters are determined at the signal-to-noise ratio equal to 10 are boldfaced.

sions for displacements and stresses caused by a uniform movement along a deepened rectangular rupture in such a half-space, was used in the calculations. To obtain the formulas, the function of the medium response to a point dislocation was integrated explicitly over the rupture area [Okada, 1985]. Input data for the program are the rupture geometry (dimensions, orientation, and depth), the amount, and direction of movement along the rupture, and the ratio of elastic moduli in the medium. Calculations yield the three-dimensional deformation field of the half-space as distributions of the displacement vector and its spatial partial derivatives.

To determine the movement parameters in the sources, we used the CMT international catalog data on the strike azimuth and dip angle of the rupture plane, the direction of the movement along the rupture, and scalar seismic moment. In addition, we included the NEIC catalog data (http://neic.usgs.gov/neis/epic_rect.html) on the moment magnitudes of earthquakes, $M_{w}$, for events $1-3$ and 5 . The moment magnitudes for events 4 and 6 , for which only the magnitudes $m_{b}$ are available in the NEIC catalog, were estimated from the $m_{b}$ dependence on $M_{w}$ reported in [Gusev and Mel'nikova, 1990].

The source dimensions: the length $L$ along the strike and the width $W$ along the dip, were estimated from the magnitude $M$, according to formulas [Riznichenko, 1976]:

$$
\begin{aligned}
& \log L=0.440 M-1.289, \\
& \log W=0.401 M-1.448 .
\end{aligned}
$$

The amount of movement along the rupture $U$ was found from the scalar seismic moment $M_{0}$ :

$$
U=M_{0} / S \mu,
$$

where $S=L W$ is the rupture area, and $\mu$ is the shear modulus of the elastic medium.
Coordinates and depths of sources for events 2-6 were specified in accordance with data of the Kamchatka regional catalog of earthquakes compiled by the KF GS RAN

(http://data.emsd.iks.ru/dbquaketxt_min/index_r.htm \#tops). We believe that the determination of the hypocenters of the Kamchatka earthquakes, based on the data from a sufficiently dense Kamchatka regional seismic network, is more reliable compared to their determination from international earthquake catalogs. For example, the difference in the hypocenter locations of earthquakes 2-6 (Table 1), determined from the Kamchatka regional catalog and from the CMT catalog amounts up to a few dozens of kilometers. The position and the rupture area of the Kronotskii earthquake (no. 1, Table 1), the strongest of the earthquakes under consideration, were refined from the cloud of aftershocks observed at the regional network within the first day after the mainshock [Levina et al., 2003].

The relative volume change, $D_{2}$, was estimated as a sum of the diagonal components of the strain tensor, which is in turn represented as the divergence of the displacement vector $\mathbf{u}$ :

$$
D_{2}=\operatorname{div} \mathbf{u}=\frac{\partial u_{x}}{\partial x}+\frac{\partial u_{y}}{\partial y}+\frac{\partial u_{z}}{\partial z},
$$

where the spatial partial derivatives of the vector $\mathbf{u}$ are calculated with the use of the Okada program mentioned above. Due to insignificant (not higher than $1 \%$ ) variations in divu in the volume containing the borehole, the value of divu at the average borehole depth $(500 \mathrm{~m})$ was assumed to be the coefficient of volumetric expansion. The resulting horizontal distributions of the volume coseismic deformation at this depth during earthquakes $1-6$ are presented in Fig. 4. Deformations in the region of borehole YuZ-5 indi- 
cated in Fig. 4 are compared in Table 1 with the data of level gage observations.

\section{DISCUSSION OF RESULTS}

\section{Comparison of the $\boldsymbol{D}_{\mathbf{1}}$ and $\boldsymbol{D}_{2}$ and Possible Errors in their Determination.}

For five of the six earthquakes, estimates of the coseismic deformation obtained with the use of these two methods coincide in sign and amplitude within an order of magnitude (Table 1.) The only exception is the earthquake of June 1, 1998 for which the sign of this estimate, based on these two methods, does not match: an extension, according to level gage observations, and a compression, according to the dislocation model. During this earthquake, the borehole turns out to be in the zone of sharp lateral variations in the deformation changing from extension to compression. In Fig. 4, such zones are seen as a crowding of contours. Insignificant changes in the input data (position and orientation of the rupture) lead to considerable changes in the resulting estimate, $D_{2}$, up to a change in sign. Thus, with a small deviation in the dip angle for the source of the June 1,1998 earthquake $\left(27^{\circ}\right.$ instead of $22^{\circ}$, according to the CMT), we obtain a quite acceptable agreement in both the value and the sign of the deformation (extension): $D_{1}=6.3 \times 10^{-9}$, according to the data on the coseismic step-like change in the water level, and $D_{2}=7.3 \times 10^{-9}$, according to the dislocation source model. The dip angle determination error in CMT is particularly noticeable at small dip angles, which is typical of interplate subduction earthquakes near a trench.

A number of simplifications implied in the model of the uniform movement along the rupture in a homogeneous half-space also reduce the accuracy considerably. Effects of sphericity and stratification become noticeable at large distances from the source; furthermore, the motion can be nonuniform in the vicinity of strong seismic events with a considerably extended source zone (for example, during the Kronotskii earthquake (no. 1, Table 1, Fig. 1)).

A certain inaccuracy is also contained in the determination of the volume coseismic deformation from the level gage observations, $D_{1}$ and, primarily, in the estimation of the tidal sensitivity of the water level, $A_{v}$. As stated above, the amplitude factors for particular waves were calculated with respect to the values of the theoretical volume tidal deformation, which can differ by up to $50 \%$ from their actual values, due to inhomogeneous structure of the medium and the distortions caused by the oceanic tide. Higher accuracy determination of $A_{V}$ would imply synchronous level gage and strain-metering observations.

The relationship between $D_{1}$ and $D_{2}$ is illustrated in Fig. 5. The points are uniformly distributed about the mean line characterizing the linear dependence between $D_{1}$ and $D_{2}$. No regularity is observed in the displacements of points with respect to each other, which indicates the absence of any systematic error in the determination of

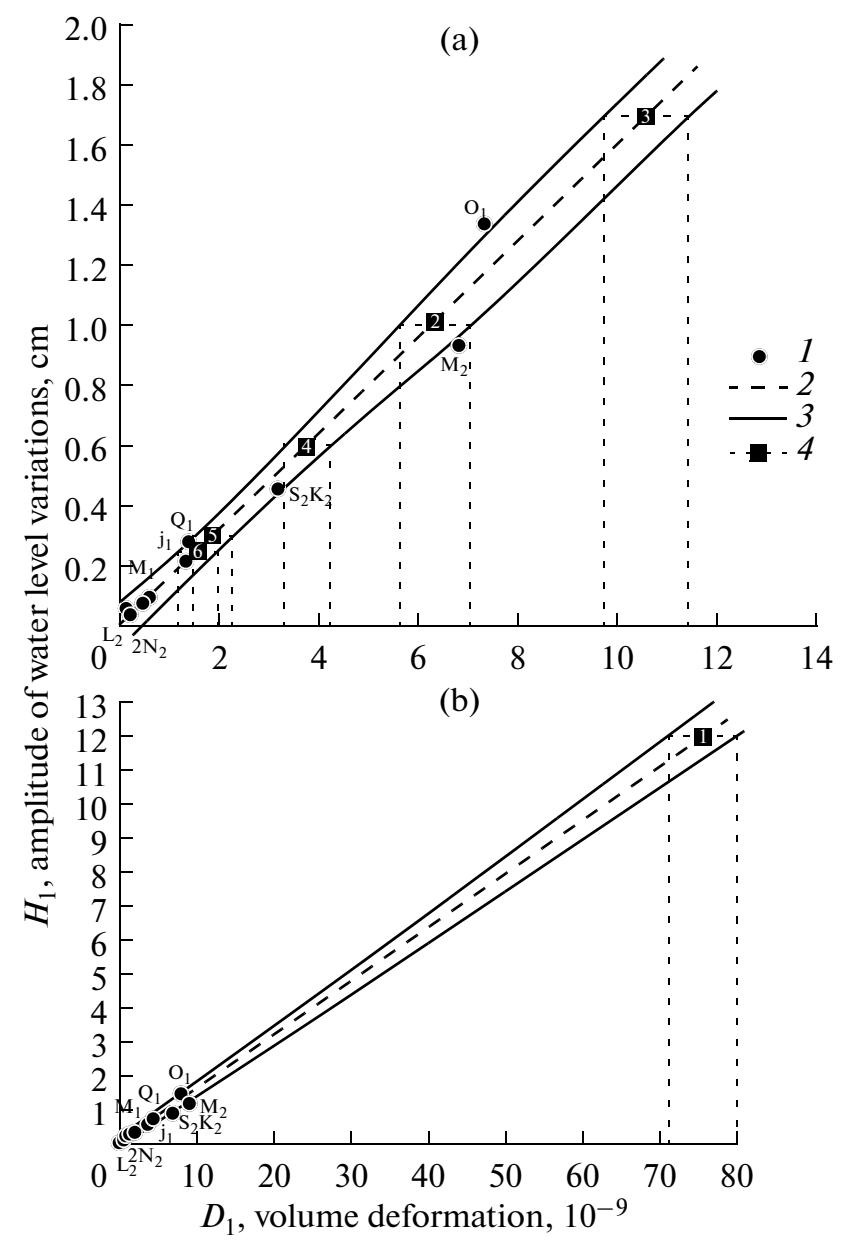

Fig. 3. (a) Dependence of tidal amplitudes identified in water level variations $H_{t}$ on the theoretical values of tidal volume deformation $D_{t}$ (Table 2): (1) tidal waves; (2) linear trend in the amplitude dependence of tidal waves in water level variations versus the tidal volume deformation for the correlation coefficient $K=0.98$; (3) the $95 \%$ confidence interval for the linear trend in the amplitude dependence of tidal waves in water level variations versus theoretical values of the tidal deformation; (4) amplitudes of coseismic water level changes during earthquakes (numerals correspond to the numbers of earthquakes in Table 1) and the ranges of the coseismic deformation estimates; and (b) estimate of the coseismic deformation during the Kronotskii earthquake (no. 1 in Table 1), linear relation between $H_{t}$ and $D_{t}$ taken into account.

coseismic deformation by both methods. Therefore, we believe that the observed discrepancies in the estimates of $D_{1} D_{2}$ are caused by errors in the estimation of volume deformations, inherent in both methods.

The Occurrence of Coseismic Step-Like Water Level Changes Dependent on the Earthquake Parameters.

The distribution of Kamchatka earthquakes with $M_{w}$ $\geq 4.5$ that occurred during the period of observations in the YuZ-5 well is presented in Fig. 6 in $M_{w}$-hypocentral distance $R$ coordinates. The specific feature of the region under study is that the strong events within the Kamchatka seismic focal zone occur mainly at hypocentral 

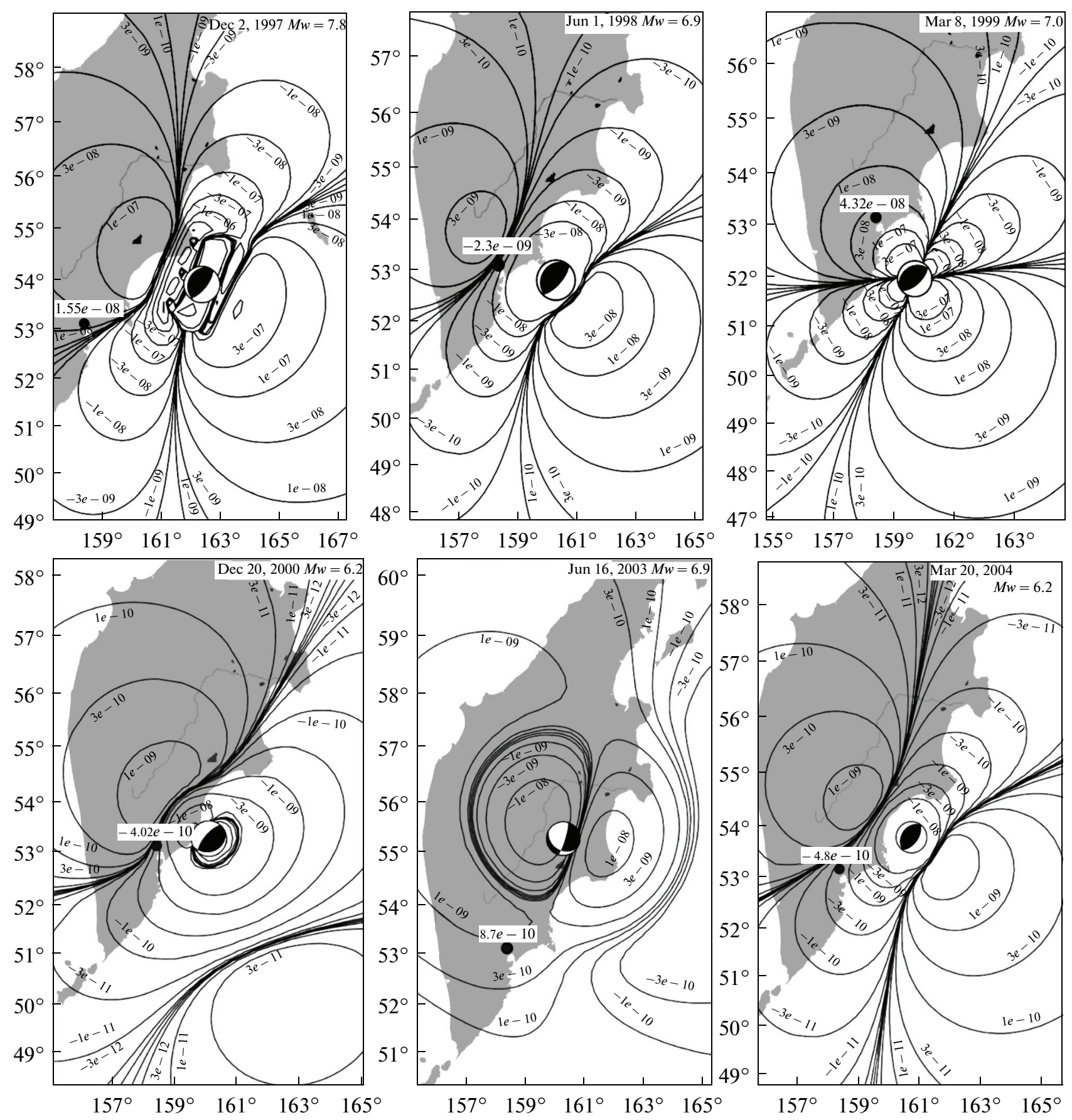

Fig. 4. Horizontal distributions of the volume coseismic deformation at the depth of $500 \mathrm{~m}$ for earthquakes $1-6$ (Table 1 ) in accordance with the dislocation source model, the values of the indicated deformation in the region of the YuZ-5 borehole (shown by the black circle).

distances of $100 \mathrm{~km}$ and larger from the YuZ-5 borehole. Earthquakes accompanied by coseismic step-like changes in the water level are the strongest and occur closest to the borehole. The parameters of these earthquakes are described, on a first approximation, by the equation $M_{w} \geq 0.004 R+5.6$, the equation of the line bordering the region of earthquakes accompanied by coseismic step changes in the water level in Fig. 6. It is just for such earthquakes that the coseismic deformation can be estimated from the level gage measurements in the Yuz-5 borehole. During the earthquakes falling below the line in Fig. 6, the amplitudes of coseismic deformations in the region of the YuZ-5 borehole are evidently smaller than units of $\times 10^{-9}$, i.e., below the strain sensitivity of the water-level variations. 
Specific Features in the Pattern of the Static Confined Response of the Water Level in the Yuz-5 Borehole During the Changes in Air Pressure and the Coseismic Deformation.

The results of cross-spectral analysis of hourly variations in the water level and atmospheric pressure reported in [Kopylova and Boldina, 2006; Kopylova, 2006] suggest the presence of a static confined response of the water level in the YuZ-5 borehole to changes in the stress-strain state of the opened reservoir in a period ranging from $6 \mathrm{~h}$ to tens of days. The static confined response of the water level is characterized by an elastic response of the pore pressure to the deformation of water-bearing rocks, which depends mainly on the elastic parameters of the controlled reservoir and is not distorted by the water flow. It was hence assumed in the work [Kopylova and Boldina, 2006] that the YuZ-5 borehole would operate as a strain recorder without substantial distortions in a period ranging from hours to a few dozens of days. At larger periods, the water-level response to changes in the stress-strain state of the groundwater reservoir can be distorted due to the processes of filtration and seasonal variations in the hydrostatic pressure. At smaller periods, the water-level response to periodic changes in the pore pressure can get weaker due to inertia in the water exchange between the borehole and the groundwater reservoir [Boldina and Kopylova, 2006].

An approximate agreement between the coseismic deformation estimates, based on the level-gage data and on the dislocation source model indicates that, first, amplitudes of coseismic water level changes adequately reflect the coseismic deformation of water-bearing rocks and, second, they are barely distorted by the inertia in the water exchange between the borehole and the groundwater reservoir. Due to this fact it becomes possible, in case of the occurrence of coseismic changes, to extend the range of the static confined response of the water level in the YuZ-5 borehole into an interval of shorter (minute scale) periods.

Here, we should take into account the fact that the varying barometric load and the coseismic deformation act upon the water-level variations in different ways. The air pressure is responsible for quasi-periodic variations in the vertical load on the groundwater reservoir controlled by the borehole, and for corresponding pore-pressure variations in this reservoir as well. With a decreasing period, the amplitudes of variations in air and pore pressure monotonically decrease. At shorter periods (minutes-a few hours) the pore-pressure response to the atmospheric load is relatively weak, and the corresponding response of the water-level in the well is suppressed (due to the nonzero water mass in the borehole shaft) by the inertial process of water exchange between the borehole and the groundwater reservoir [Boldina and Kopylova, 2006]. The maximum and constant value of the amplitude transfer function conveying the air pressure variations to the water-level variations is observed in the YuZ-5 borehole only at periods of $\geq 6 \mathrm{~h}$.

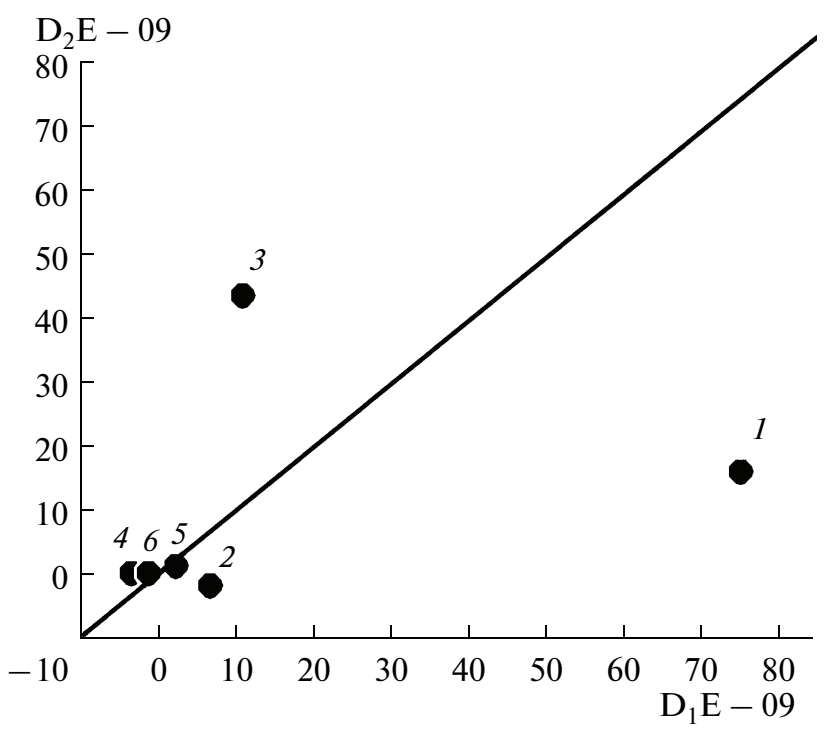

Fig. 5. Relationship between the coseismic volume deformation estimated from the level gage observations $\left(D_{1}\right)$ and from the model of dislocation source $\left(D_{2}\right)$. Numerals correspond to the numbers of earthquakes in Table 1.

When a rupture is formed in the source of a strong earthquake, a rapid redistribution of the stress-strain state of water-bearing rocks occurs, involving the entire volume of rocks. The change in the pore-pressure in the groundwater reservoir (a decrease on extension and an increase on compression) instantaneously reaches its maximum. In this case, the borehole water level response to the pore-pressure pulse is hardly distorted by the inertial character of the water exchange between the borehole and the groundwater reservoir, because the entire volume

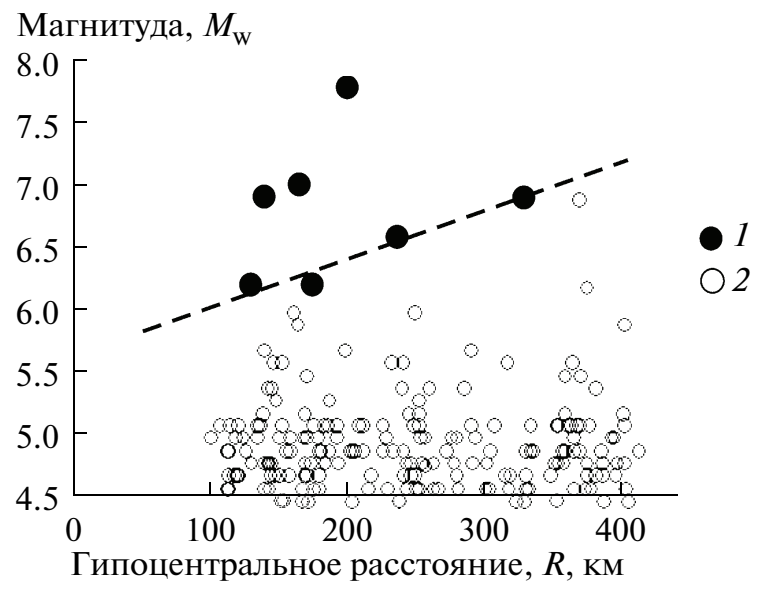

Fig. 6. Distribution of earthquakes with $M_{w} \geq 4.5$ that occurred during observations in the YuZ-5 borehole in 1997-2004 as a function of the earthquake parameters: magnitude $M_{w}$ and hypocentral distance $R$, km: (1) earthquakes accompanied by coseismic changes in the water level (Table 1); (2) earthquakes for which no coseismic changes in the water level were observed. 
of interrelated water-bearing rocks controlled by the borehole now "works" for the maximum release or absorption of water, which is facilitated by favorable conditions in the YuZ-5 borehole: a long (490 m) open shaft connecting the borehole and the groundwater reservoir, and a large surface of the shaft contact with the adjacent water-bearing rocks $\left(258.6 \mathrm{~m}^{2}\right)$.

\section{CONCLUSIONS}

(1) A new method for the point assessment of the volume coseismic deformation arising during the formation of ruptures in the sources of strong $\left(M_{w} \geq 6\right)$ Kamchatka earthquakes is presented in this work. The method makes use of the water level observations in wells. The proposed technique for estimating the coseismic deformation is based on the results provided by complex analysis of barometric and tidal responses of the water level in the well, and on the amplitude data of coseismic water level changes measured with $10 \mathrm{~min}$ time resolution.

Analysis of the water level barometric response makes it possible to reveal the undistorted static confined barometric response of the water level to the changes in the stress-strain state of the controlled groundwater reservoir and to determine the frequency range of this response. Tidal sensitivity of the water level is estimated from the analysis of tidal variations in the water level and is used as a normalizing coefficient in estimating the coseismic deformation from amplitudes of recorded coseismic water level changes during the formation of ruptures in the sources of earthquakes.

Through the example of six Kamchatkan earthquakes, it is shown that the estimates of coseismic deformations provided by the water level observations in the YuZ-5 well, in most cases, agree both in sign and in amplitude with the theoretical estimates calculated in accordance with the model of an extended source of dislocation. A certain difference in the values of coseismic volume deformation provided by the two methods is likely due to the errors inherent in both methods.

(2) Detection of coseismic water level changes in the YuZ-5 well during strong local earthquakes indicates the presence of static confined conditions in the boreholereservoir system at minute periods during the formation of seismic ruptures and pulsed changes in the stress-strain state of water-bearing rocks. The extended open part of the YuZ-5 borehole shaft and a large contact surface of the shaft and adjacent water-bearing rocks are factors that favor the occurrence of such a response. The parameters of those Kamchatka earthquakes (the ratios of the magnitude to hypocentral distance), which can be accompanied by coseismic deformations with amplitudes of $\times 10^{-9}$ units and higher in the region of the YuZ-5 borehole are described by the equation $M_{w} \geq 0.004 R+5.6$.

The agreement between theoretical estimates of the coseismic deformation and its experimental estimates obtained from the water level observations confirms the strain sensitivity of the water level in the YuZ-5 well and augurs well for its prospects of being used in the future in the system of geophysical monitoring in Kamchatka.

\section{REFERENCES}

1. S. V. Boldina and G. N. Kopylova, "Estimation of the Inertial Effect of Water Exchange between a Borehole and a Groundwater Reservoir," in Vestnik KRAUNTs, Series Earth Sciences, No. 2, Iss. 8, 112-119 (2006) [in Russian].

2. A. A. Gusev and B. N. Mel'nikova, "Relations between Magnitudes-World-Average and for Kamchatka," Vulkanol. Seismol., No. 6, 55-63 (1990).

3. I. G. Kissin, Hydrogeological Monitoring of the Earth's Crust," Fizika Zemli, No. 8, 58-69 (1993).

4. G. N. Kopylova, "Water-Level Variations in Borehole YuZ-5, Kamchatka, Caused by Earthquakes," Vulkanol. Seismol., No. 6, 52-64 (2006).

5. G. N. Kopylova and S. V. Boldina, "Estimation of Poroelasic Parameters of a Groundwater Reservoir (from Data of Level-Gauge Observations in Borehole YuZ-5, Kamchatka)," Vulkanol. Seismol., No. 2, 17-28 (2006).

6. P. Melchior, The Earth Tides (Oxford, 1966; Mir, Moscow, 1968).

7. Y. Okada, "Surface Deformation Due to Shear and Tensile Faults in a Half-Space," Bull. Seism. Soc. Amer. 75, 1135-1154 (1985).

8. Yu. V. Riznichenko, "Dimensions of a Crustal Earthquake Source and Seismic Moment," in Investigations in the Physics of Earthquakes (Nauka, Moscow, 1976) [in Russian].

9. E. A. Roeloffs, "Hydrologic Precursors to Earthquakes: A Review,” Pure Appl. Geophys. 126, 177-209 (1988).

10. S. Rojstaczer, "Intermediate Period Response of Water Levels in Wells to Crustal Strain: Sensitivity and Noise Level,” J. Geophys. Res. 93, 13619-13634 (1988).

11. S. Rojstaczer and D. S. Agnew, "The Influence of Formation Material Properties on the Response of Water Levels in Wells to Earth Tides and Atmospheric Loading," J. Geophys. Res. 94, 12403-12411 (1989).

12. H. Wakita, "Water Levels as Possible Indicators of Tectonic Strain," Science, No. 189, 553-555 (1975).

13. H. G. Wenzel, "Earth Tide Analysis Package ETERNA 3.0,” BIM, No. 118, 8719-8721 (1994).FIGURE CAPTIONS 